\title{
RED INALÁMBRICA DE BIOSENSORES ENZIMÁTICOS PARA LA DETECCIÓN DE CONTAMINANTES EN AGUAS
}

\author{
Duvan Fernando Morales Castaño', Juan Bernardo Cano Quintero², Nelson Londoño Ospina ${ }^{3}$ \\ ${ }^{1}$ Ing. Electricista, Candidato a Magister en Ingeniería de la Universidad de Antioquia. \\ ${ }^{2} \mathrm{PhD}$ en Ingeniería Electrónica, Docente Universidad de Antioquia. \\ ${ }^{3} \mathrm{PhD}$ en Robótica, Docente Universidad de Antioquia.
}

Grupo manejo eficiente de la energía (GIMEL). Medellín - Colombia

Email: duvan.morales@udea.edu.co,bernardo.cano@udea.edu.co,nelson.londono@udea.edu.co

\begin{abstract}
RESUMEN
En este artículo se presenta el desarrollo de una red inalámbrica de biosensores amperimétricos, que podrá ser distribuida en las fuentes de agua potable, con el fin de alertar sobre la presencia de contaminantes tóxicos. Este sistema no reemplazará los métodos tradicionales para la detección de contaminantes, pero tiene la ventaja de ser un sistema de bajo costo, ideal para aplicaciones en campo y donde sea necesario el procesamiento de una gran cantidad de muestras. se pudo concluir que los biosensores no tienen una respuesta lineal a la concentración del analito, por lo que su uso es cualitativo y no cuantitativo. Se presentan resultados del desarrollo de los circuitos electrónicos necesarios para la transducción amperométrica, los circuitos para el procesamiento de datos y la comunicación inalámbrica.
\end{abstract}

Palabras clave: Biosensor, amperometría, red inalámbrica, alertas tempranas, sensores.

Recibido: 29 de Marzo de 2019. Aceptado: 20 de Julio de 2019

Received: March 29, 2019 Accepted: July 20, 2019

\section{ENZYMATIC BIOSENSOR WIRELESS NETWORK FOR DETECTION OF WATERS CONTAMINANTS}

\begin{abstract}
This article presents the development of an amperometric biosensor wireless network, which can be distributed in drinking water sources, in order to warn about toxic contaminants presence. This system will not replace traditional contaminants detection methods, but it has the advantage of being a low cost system, ideal for field applications and where the processing of a large number of samples is necessary. it can be concluded that the biosensors do not have a linear response to the analyte concentration, so their use is qualitative and not quantitative. The results of the development of the electronic circuits for the amperometric transduction, the circuits for data processing and wireless communication are presented.
\end{abstract}

Keywords: Biosensor, amperometry, wireless network, early alerts, sensors.

Cómo citar este artículo: D. Morales, J. Cano, N. Londoño. "Red inalámbrica de biosensores enzimáticos para la detección de contaminantes en aguas", Revista Politécnica, vol. 15, no.29 pp.09-22, 2019. DOI: 10.33571/rpolitec.v15n29a1 


\section{INTRODUCCIÓN}

La detección de contaminantes en las fuentes de agua es prioridad para las plantas de potabilización, debido a que su consumo puede ser perjudicial para la salud humana, por lo que cada vez cobra mayor importancia evaluar in situ, la posible presencia de contaminantes en las principales fuentes de agua que abastecen las plantas de potabilización, las cuales provienen, en su gran mayoría, de las zonas rurales, donde las prácticas agrícolas utilizan pesticidas para el control de plagas en los cultivos. Estos pesticidas pueden llegar a diluirse en los ríos y ser consumido por las personas, afectando la salud de la población.

Los biosensores son dispositivos que utilizan un elemento biológico sensible, para obtener información del ambiente que los rodea. Este elemento, conocido como biomediador o biorreceptor, puede estar conformado por organelos celulares, células completas, enzimas, anticuerpos, etc., que tengan la capacidad de cambiar su comportamiento, como reacción a un agente externo. Dicho cambio de comportamiento se representa como el cambio de una de sus propiedades fisicoquímicas como puede ser la temperatura, la masa, las propiedades ópticas (fluorescencia, índice de refracción, luminiscencia, etc.), las propiedades eléctricas (corriente, resistencia, impedancia). Los cambios de estas variables pueden ser analizados utilizando un sistema de transducción adecuado (figura 1).

Muchas de las aplicaciones de las tecnologías biosensoristicas (biosensing technologies) [1], van desde la detección de contaminantes de todo tipo, sea de especies químicas o bactericas [2-4], en fuentes de agua dulce [4] y marinas [6,7], en la vigilancia del ecosistemas [8-16], agroalimentación [17], en la optimización de antioxidantes [18,19], detección de problemas endocrinológicos [20], llegando hasta la parte espacial [21-22].

Es importante tener claro que, el desarrollo de biosensores implica un trabajo de especialistas en diferentes áreas del conocimiento; ósea que es una de las áreas más multidisciplinares que existen ya que necesitan de especialistas en la química y la biología, necesarios para la selección, crecimiento y estabilización del material biológico [23], así como el análisis de sus interacciones con el analito; hasta ingenieros electrónicos, mecánicos e informáticos [24-29], para el diseño de sistemas de transducción y acondicionamiento de las señales, diseño de los sistemas de toma de muestras y el análisis de datos.

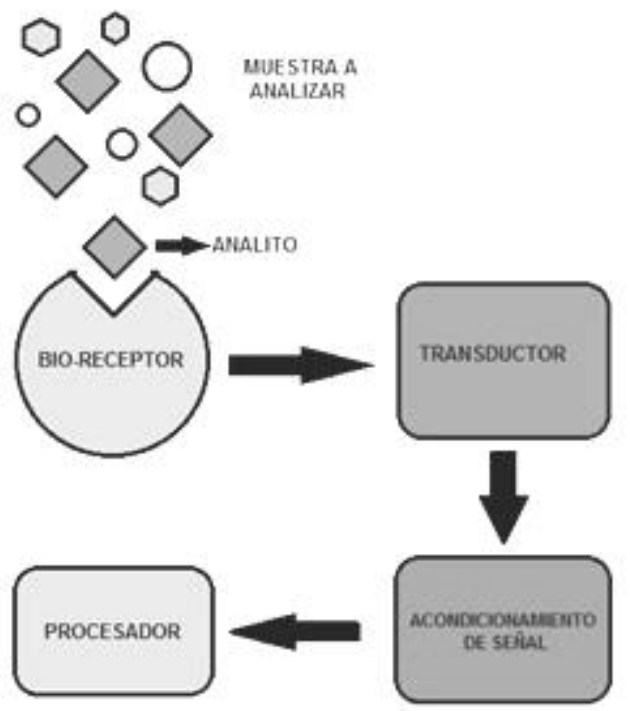

Fig. 1. Esquema de un biosensor.

Diferentes biosensores han sido reportados para la detección de plaguicidas y herbicidas en todo tipo de aguas (dulces y marinas). Enzimas como la acetilcolinesterasa, la tirosinasa y la lacasa (entre otras) han sido empleadas en biosensores amperométricos, permitiendo la detección de diferentes tipos de plaguicidas [30].También se han reportado biosensores que emplean detección óptica (fluorescencia) y organismos fotosintéticos para esta aplicación [31]. En estos trabajos se enfatiza la importancia de los biosensores como elementos de pre-screening y elementos de alerta temprana (early warning) [32] es decir, elementos viables para obtener una medida rápida y de bajo costo en campo, principalmente con el objeto de proporcionar alarmas de presencia de contaminantes.

Por otro lado, una red inalámbrica de sensores (Wireless Sensor Network, WSN), es una red con numerosos dispositivos distribuidos espacialmente, que utilizan sensores para monitorear condiciones físicas 0 ambientales. Los dispositivos son unidades autónomas llamados nodos, los cuales tienen como tarea principal recolectar datos. Debido a que los nodos pueden ser ubicados en zonas remotas o de difícil acceso, cada nodo necesita consumir la menor cantidad de energía posible para asegurar la máxima autonomía [33]; así, el acceso a estas zonas no deberá ser recurrente, evitando sobrecostos en su 
mantenimiento. Un nodo generalmente es un sistema basado en un microcontrolador que incorpora un módulo de radio frecuencia (RF), un sensor y un sistema de alimentación eléctrica [34].

Además de los nodos, la red inalámbrica de sensores incorpora un Gateway, el cual es un dispositivo que provee conectividad entre los nodos distribuidos y alguna red superior por ejemplo internet (figura 2). El protocolo inalámbrico usado en las WSN depende de los requerimientos de la aplicación. Algunos de los estándares disponibles incluyen radios de $2.4 \mathrm{GHz}$, basados en los estándares IEEE 802.15.4 o IEEE 802.11 (WiFi), Redes de telefonía celular, entre otros. [35]

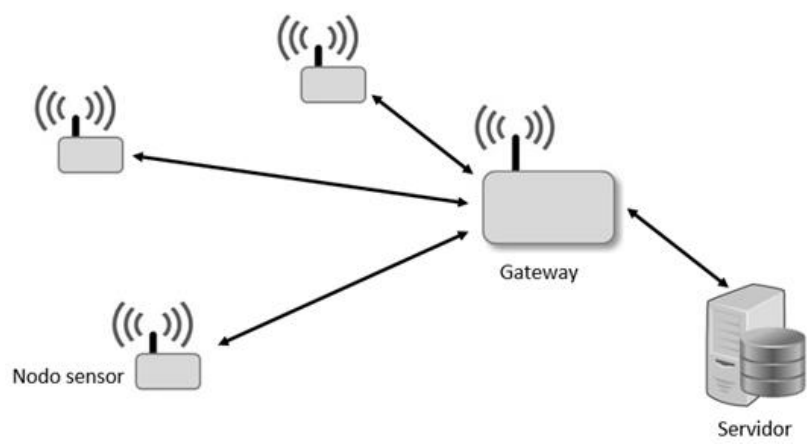

Fig. 2. Estructura de una red inalámbrica de sensores

Algunas de las aplicaciones en las que se utilizan las redes inalámbricas de sensores son: la medición de la polución atmosférica [36], monitoreo de volcanes [37], la agricultura [38], domótica [39], entre otros.

En este trabajo, se presenta el desarrollo electrónico de una red inalámbrica de biosensores enzimáticos, que emplea transducción amperométrica y permite el uso simultáneo de múltiples biomediadores (enzimas), para la detención de pesticidas en fuentes de agua potable, utilizando un sistema de comunicación inalámbrica para el envío de los datos obtenidos y sus posibles alarmas.

La red inalámbrica permitirá ubicar los biosensores en zonas remotas, dando la posibilidad de detectar los contaminantes antes de que lleguen a la planta de potabilización, permitiendo tomar acciones preventivas. Además, la modularidad de la red permite adaptarla e integrar otros tipos de detección y/o biomediadores, de una forma simple.
Es importante anotar que, el uso de biosensores no sustituirá las técnicas convencionales de detección de contaminantes, ya que los biosensores no alcanzan los niveles de precisión, exactitud y selectividad que caracterizan a los equipos de laboratorio; pero, al detectar la presencia de contaminantes con un dispositivo de bajo costo, permite disminuir el número de muestras que deben ser enviadas a laboratorios, para la realización de análisis profundos, enviando solo aquellas que dan positivo en la detección del biosensor; lo que económicamente se traduce en una reducción de los costos de monitorización de las fuentes de agua.

\section{MÉTODOS}

El desarrollo de la red inalámbrica de biosensores se divide en tres partes principales: El diseño del transductor amperométrico, el diseño de la unidad central de procesamiento y la implementación de la comunicación inalámbrica. Las dos primeras partes conforman el nodo de medida y son las encargadas del análisis de las aguas.

\section{Transductor amperométrico}

La amperometría consiste en medir la corriente generada en el proceso de oxido/reducción de una sustancia electroactiva, en contacto con un sistema de electrodos. Para hacer posible este proceso, se debe someter los electrodos a una diferencia de potencial, que dependerá de las características de la reacción y del material de los electrodos. Las corrientes generadas por este tipo de biosensores son del orden de nanoamperios y microamperios, por esta razón es necesario un adecuado sistema electrónico para su medida.

El análisis de las corrientes medidas varia con el tipo de biomediador. En un biosensor enzimático, se genera una corriente que se incrementa hasta alcanzar un estado estable, al entrar en contacto con el analito, se evidencia una reducción en la corriente (figura 3 ). La reducción de la corriente puede ser usada para estimar la concentración del analito. Normalmente dicha reducción se presenta como un porcentaje de inhibición respecto a la corriente inicial. 


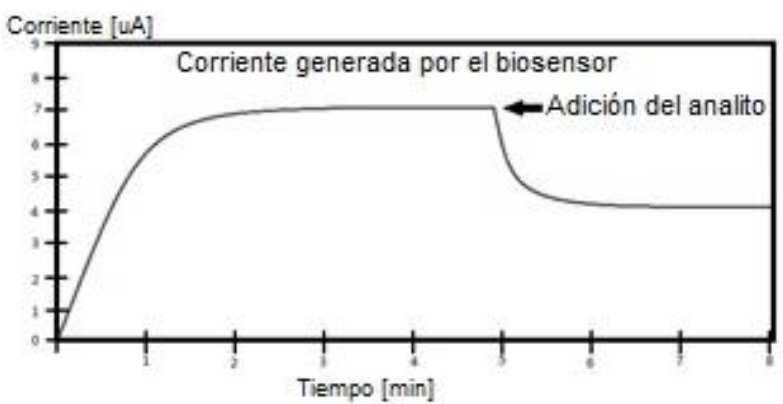

Fig. 3. Respuesta en corriente de la prueba de amperometría.

Comúnmente se utiliza un sistema de tres electrodos compuesto por un electrodo de referencia (RE), un electrodo auxiliar (CE) y un electrodo de trabajo (WE) (figura 4). En esta configuración el electrodo auxiliar y el electrodo de trabajo están sometidos a una diferencia de potencial. El electrodo de referencia presenta una alta impedancia, por lo que el flujo de corriente a través de él es cero (Ire $=0$ ). Este electrodo es usado para realimentar el sistema que controla el potencial entre el electrodo auxiliar y el electrodo de trabajo. La corriente generada en el proceso de amperometría (Icw), es medida a través del electrodo de trabajo.

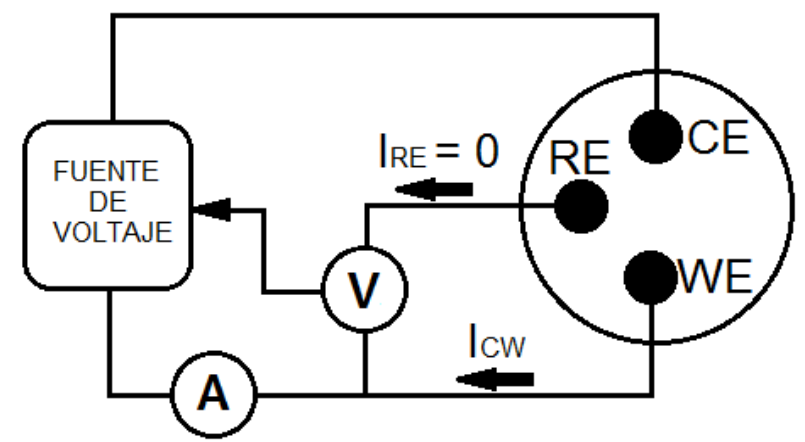

Fig. 4. Sistema de tres electrodos usado en amperometría.

Los sistemas de electrodos para biosensores son implementados normalmente en S.P.E. (ScreenPrinted Electrodes) (figura 5). Estos son construidos depositando diferentes capas conductoras (carbón, oro, platino, plata), sobre una base de PVC, cerámica o aluminio. Poseen una línea de contacto para conectarse con el circuito transductor. Por ser elementos destinados a ser desechados, los S.P.E tienen la ventaja de ser de bajo costo, de tamaño pequeño y son producidos en masa.

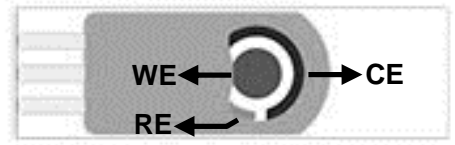

Fig. 5. Sistema de electrodos S.P.E de la empresa DropSens [41]

El circuito encargado de mantener el potencial requerido en el sistema de electrodos se conoce como potenciostato (figura 6). El amplificador operacional IC8B, se utiliza para imponer una alta impedancia en el electrodo de referencia (RE) y garantizar que la corriente que circula por este sea nula. Este amplificador está configurado como seguidor de voltaje y su salida (Vsense) es utilizada para monitorear el potencial de referencia. Esto permite detectar situaciones de falla del potenciostato y entregar las correspondientes alarmas.

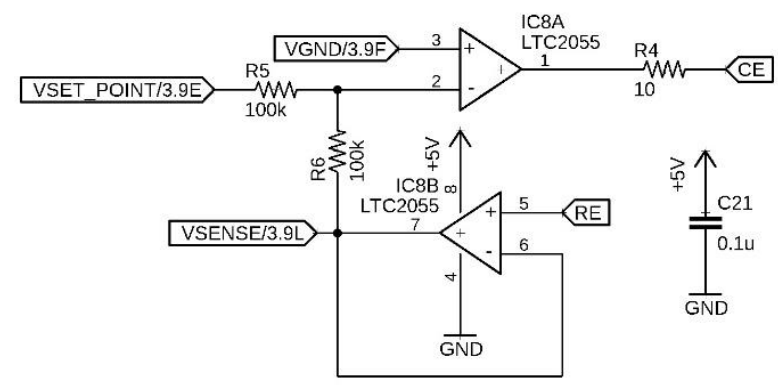

Fig. 6. Circuito Potenciostato

La entrada (Vset_point) recibe el potencial deseado desde un conversor digital análogo. Si el amplificador IC8A, configurado como amplificador inversor de ganancia unitaria, está trabajando en su región lineal, el voltaje en el electrodo de referencia (RE) es de igual magnitud y de signo opuesto a la señal Vset_point.

La operación adecuada del potenciostato depende, en gran medida, de las condiciones de las sustancias en contacto con los electrodos. Una mala conductividad entre CE y RE y/o una corriente excesiva a través de WE, requerirá un mayor voltaje entre $C E$ y $R E$ que puede poner los amplificadores operacionales en saturación.

El circuito potenciostato es implementado empleando los amplificadores operacionales LTC2055HV, debido a su bajo offset de entrada, baja corriente de polarización de entrada y bajo ruido. 
Las características de este amplificador se muestran en la tabla 1.

Tabla 1. Características amplificador operacional

\begin{tabular}{|ll|}
\hline \multicolumn{2}{|c|}{ LTC2055HV } \\
\hline Input Offset Voltaje & $\pm 0.5 \mu \mathrm{V}$ \\
Input Bias Current & $\pm 3 \mathrm{pA}$ \\
Input Offset Current & $\pm 6 \mathrm{pA}$ \\
Input Noise Voltage & $1.6 \mu \mathrm{Vp}-\mathrm{p}$ \\
Common Mode Rejection Ratio & $130 \mathrm{~dB}$ \\
\hline
\end{tabular}

El circuito utilizado para medir la corriente generada en el proceso de amperometría consta de un amplificador operacional en configuración de amplificador de trans-resistencia. Su entrada inversora está conectada directamente al electrodo de trabajo (WE), lo que garantiza un potencial cercado a cero en este electrodo (necesario para la precisión del potencial de referencia). Este amplificador entrega a su salida un voltaje proporcional a la corriente que circula por él (figura 7).

La constante de proporcionalidad del circuito está dada por la resistencia de retroalimentación en el amplificador operacional y su valor es de vital importancia para garantizar un rango apropiado de señal. Para aumentar la flexibilidad del circuito, permitiendo medir diferentes rangos de la corriente de entrada, se implementa un sistema de multiplexado, para cambiar la resistencia de retroalimentación a elección del usuario. El multiplexor permite seleccionar cuatro diferentes valores de resistencia de retroalimentación.

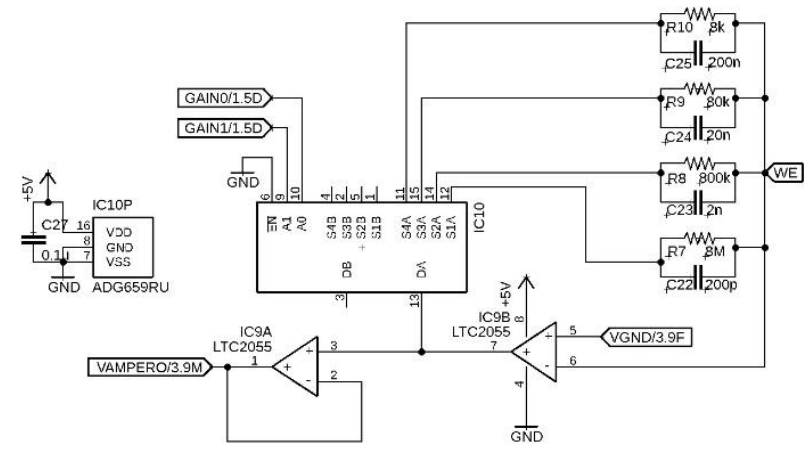

Fig. 7. Circuito medidor de corriente

El circuito integrado IC9B es el amplificador de trans-resistencia y el circuito integrado IC10 es el multiplexor. Las resistencias $R 7, R 8, R 9$ y $R 10$ son las encargadas de definir la ganancia del circuito $y$ a cada una de ellas, se le asocia un capacitor en paralelo por razones de filtrado y estabilidad del amplificador. Las señales Gain0 y Gain1 son digitales y permiten seleccionar la resistencia de retroalimentación.

Tabla 2. Rangos de medida de corriente.

\begin{tabular}{|llll|}
\hline Gain1 & Gain0 & $\mathrm{R}[\Omega]$ & Rango \\
\hline 0 & 0 & $8 \mathrm{M}$ & $-70 \mathrm{nA}$ a 70nA \\
0 & 1 & $800 \mathrm{k}$ & $-700 \mathrm{nA}$ a $700 \mathrm{nA}$ \\
1 & 0 & $80 \mathrm{k}$ & $-7 \mu \mathrm{A}$ a $7 \mu \mathrm{A}$ \\
1 & 1 & $8 \mathrm{k}$ & $-70 \mu \mathrm{A}$ a $70 \mu \mathrm{A}$ \\
\hline
\end{tabular}

Como se observa en la tabla 2, el circuito medidor de corriente trabaja con señales de muy bajo nivel (del orden de los nA y $\mu A$ ); por esta razón, se deben utilizar circuitos electrónicos que no afecten la medida. En el caso de los amplificadores operacionales, deben tener una corriente de polarización de sus entradas baja, ya que la corriente absorbida por las entradas del amplificador, pueden aumentar o disminuir la magnitud de la corriente entregada por el biosensor, produciendo errores en la medida. Para la implementación del medidor de corriente se emplea el amplificador LTC2055HV, el cual tiene una corriente de polarización de sus entradas de $\pm 3 p A$. Esta corriente no es significativa para los rangos de corriente en los cuales trabaja el circuito medidor de corriente. El multiplexor empleado para la selección de rango también puede afectar la medida de la corriente. Los principales problemas generados por el multiplexor son: la inyección de carga desde las entradas de selección hacia la salida del multiplexor y las corrientes de fuga provenientes de los canales inactivos. El multiplexor usado para el circuito de medida es el ADG659RU. Sus características de corrientes de fuga e inyección de carga se muestran en la tabla 3.

Tabla 3. Características del multiplexor.

\begin{tabular}{|lc|}
\hline \multicolumn{2}{|c|}{ ADG659 } \\
\hline Leakage Currents & $\pm 5 \mathrm{pA}$ \\
Charge injection & $2 \mathrm{pC}$ \\
\hline
\end{tabular}

Todas las señales análogas provenientes del circuito potenciostato y del circuito medidor de corriente, pasan a través de un conversor análogo digita (ADC) tipo Sigma Delta con resolución de 
hasta 24 bits. Este tipo de ADC dispone de un filtro digital, que permite tener un rechazo simultaneo de señales a $50 \mathrm{~Hz}$ y $60 \mathrm{~Hz}$ (como las provenientes de las redes eléctricas), principal componente de ruido en muchos sistemas sensoriales.

Las señales digitales son procesadas por un microcontrolador, en cual es el encargado del control del transductor amperométrico y de el envío de la información a la unidad central de procesamiento. El diagrama de bloques del transductor amperométrico (figura 8) consta de 5 bloques: microcontrolador, conversor análogo digital, conversor digital análogo, potenciostato y medidor de corriente.

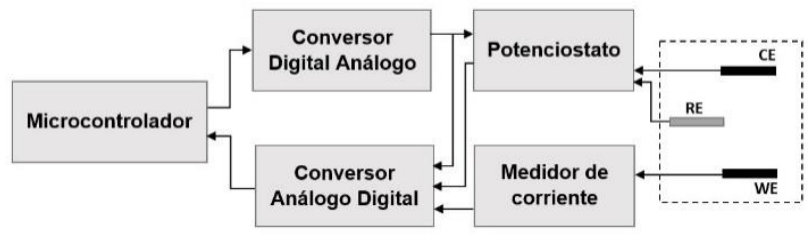

Fig. 8. Diagrama de bloques transductor amperométrico.

\section{Unidad central de procesamiento}

La unidad de control es un circuito electrónico utilizado como dispositivo maestro en el nodo de medida. Esta unidad se encarga del manejo de los transductores amperométricos, indicándoles cuando iniciar una prueba de amperometría. Además, se encarga de la recopilación de los datos entregados por los transductores y su análisis, para determinar si existe 0 no presencia de contaminantes tóxicos. Una vez obtenidos los resultados de las pruebas, la unidad de control genera archivos con reportes de los resultados y los envía hacia un servidor central, donde podrán ser observados por el personal correspondiente. En caso de detectar contaminantes tóxicos, la unidad de control envía alarmas al centro de monitoreo para alertar sobre la presencia de estos.

Debido a su capacidad de cómputo, se utiliza una computadora de placa reducida o SBC (Single Board Computer), como unidad de control. Un SBC es una tarjeta electrónica que contiene todos o la mayor parte de los componentes de una computadora, integrados dentro de la misma placa base. La principal característica de las SBC son sus reducidas dimensiones y su bajo costo. Debido a los grandes niveles de integración y reducción de componentes y conectores, los computadores de placa reducida suelen ser más pequeños, livianos, más confiables y con un mejor manejo de la potencia eléctrica que los computadores de múltiples tarjetas. Para este proyecto, se optó por la SBC Raspberry Pi.

Raspberry Pi es un SBC desarrollado en el Reino Unido por la Fundación Raspberry PI [40]. Sus últimas dos versiones son: la Raspberry $\mathrm{Pi} 3 \mathrm{~B}+\mathrm{y}$ la Raspberry Pi Zero W.

La Raspberry $\mathrm{Pi} 3 \mathrm{~B}+$ contiene un procesador de cuatro núcleos con arquitectura de 64bits, frecuencia de $1.2 \mathrm{GHz}$ y una memoria de $1 \mathrm{~GB}$. La Raspberry Pi Zero por su parte, cuenta con un procesador mono núcleo de 32bits que corre a una frecuencia de $1 \mathrm{GHz}$ y una memoria RAM de 512MB. Ambas versiones se caracterizan por tener conexión WiFi y Bluetooth.

La fundación Raspberry $\mathrm{Pi}$ tiene un sistema operativo oficial llamado Raspbian (unión de las palabras Raspberry y Debian). Este sistema operativo Linux ofrece dos versiones: Raspbian with desktop y Raspbian Lite. La gran diferencia de los dos sistemas operativos es la interfaz gráfica. La versión Raspbian Lite no cuenta con interfaz gráfica y no tiene instalados los programas que requieran de esta para funcionar. Por esta razón, el sistema operativo Raspbian Lite necesita menos memoria para su instalación y menos requisitos de hardware.

Aunque la Raspberry Pi Zero $\mathrm{W}$ tiene menos potencia computacional, se destaca por su tamaño reducido (siendo este casi un tercio del tamaño de la Raspberry $\mathrm{Pi}$ 3B) y su menor consumo de energía. Después de realizar pruebas de consumo energético a la Raspberry Pi 3B y la Raspberry Pi Zero W, corriendo las dos versiones de Raspbian, se determina que la mejor opción es usar la Raspberry Pi Zero $\mathrm{W}$ con el sistema operativo Raspbian Lite. Las pruebas se pueden observar en la sección de resultados.

\section{Comunicación inalámbrica}

Para la transmisión de datos desde el nodo de medida hasta el servidor central, se pueden usar diferentes tecnologías de transmisión inalámbrica como WiFi y GSM/GPRS. 
WiFi: En redes de biosensores de corto alcance, donde sus nodos no sobrepasan los $100 \mathrm{~m}$ de separación con el Gateway y para pruebas de laboratorio, es posible usar la tecnológica WiFi (figura 9). Para transmitir datos por WiFi, se utiliza el módulo que trae incorporado la Raspberry $\mathrm{Pi}$ Zero W y un router WiFi como Gateway. El Gateway debe estar conectado a la red de internet o directamente al servidor central.

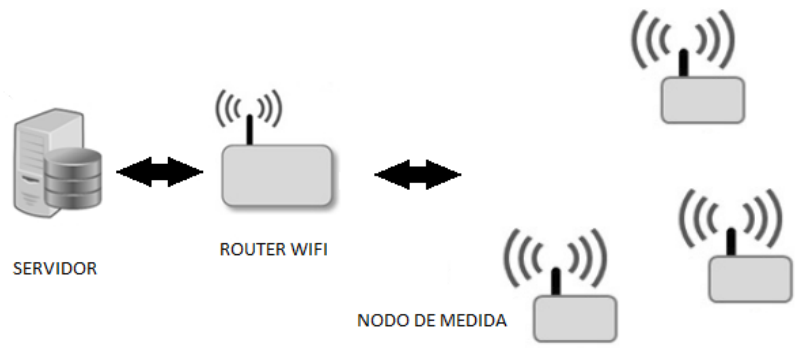

Fig. 9. Esquema de red con tecnología WiFi.

GSM/GPRS: para redes de largo alcance la tecnología WiFi no es suficiente. La tecnología GSM/GPRS se apoya en la red celular para la transmisión y recepción de datos (figura 10). Para conectar el nodo de medida a la red celular, se necesita adicionar un módulo como el A6 de la empresa Ai-thinker.
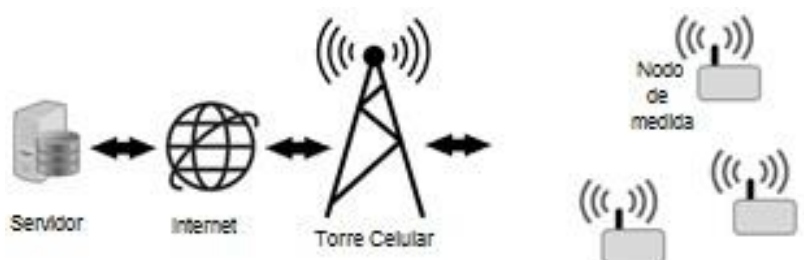

Fig. 10. Esquema de red con tecnología GSM/GPRS.

\section{RESULTADOS}

\section{Transductor amperométrico}

Se realizó el diseño de una tarjeta de circuito impreso (PCB, printed circuit board) para la implementación física del circuito amperométrico (figura 11 y 12). El diseño tuvo como objetivo principal una alta integridad de las señales a medir, debido a su bajo nivel (señales del orden de los $\mathrm{mV}, \mu \mathrm{A}$ y $\mathrm{nA}$ ). También se tuvo en cuenta obtener un circuito de dimensiones reducidas para facilitar su uso en campo.

La PCB fue diseñada a cuatro capas, lo que garantiza la existencia de planos de tierra y de alimentación sin cortes. Se realizó una cuidadosa partición de las secciones análogas y digitales para evitar interferencias.

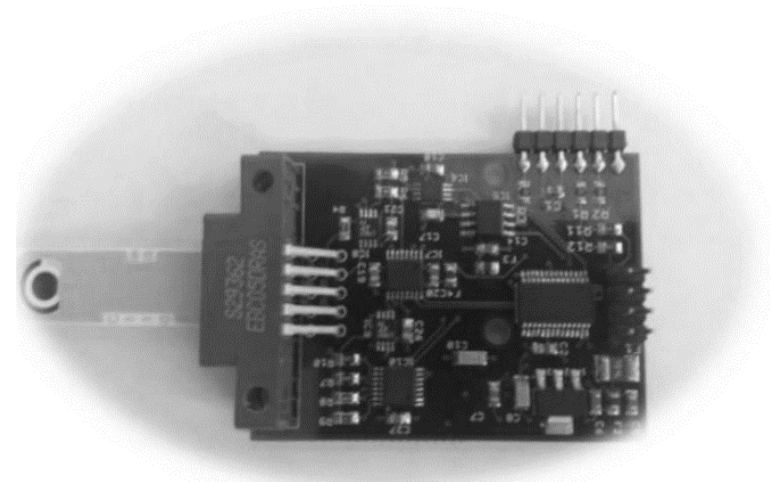

Fig. 11. PCB vista superior con el sistema de electrodos S.P.E. conectado.

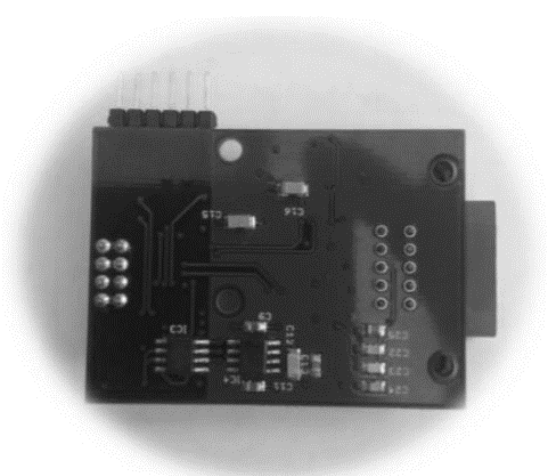

Fig. 12. PCB vista inferior.

Se realizó la calibración de los diferentes circuitos del transductor amperométrico para verificar su correcto funcionamiento.

La calibración del conversor digital análogo se realizó enviando diferentes valores binarios al circuito integrado. Usando un voltímetro se midió el voltaje a la salida del DAC y se comparó con el valor teórico. Los resultados se muestran en la tabla 4 y la figura 13. 
Tabla 4. Calibración conversor digital análogo.

\begin{tabular}{|llll|}
\hline $\begin{array}{c}\text { DAC } \\
\text { Código } \\
\text { de } \\
\text { entrada }\end{array}$ & $\begin{array}{c}\text { Valor } \\
\text { teórico de } \\
\text { la salida } \\
\text { [mV] }\end{array}$ & $\begin{array}{c}\text { Valor } \\
\text { real de } \\
\text { la salida } \\
\text { [mV] }\end{array}$ & $\begin{array}{c}\text { Error } \\
{[\mathrm{mV}]}\end{array}$ \\
\hline 0 & -1024 & $-1019,7$ & 4,3 \\
400 & -824 & $-820,7$ & 3,3 \\
800 & -624 & $-620,8$ & 3,2 \\
1200 & -424 & $-420,3$ & 3,7 \\
1600 & -224 & $-220,5$ & 3,5 \\
2000 & -24 & $-20,4$ & 3,6 \\
2400 & 176 & 179,3 & 3,3 \\
2800 & 376 & 379 & 3 \\
3200 & 576 & 579,5 & 3,5 \\
3600 & 776 & 779,3 & 3,3 \\
\hline
\end{tabular}

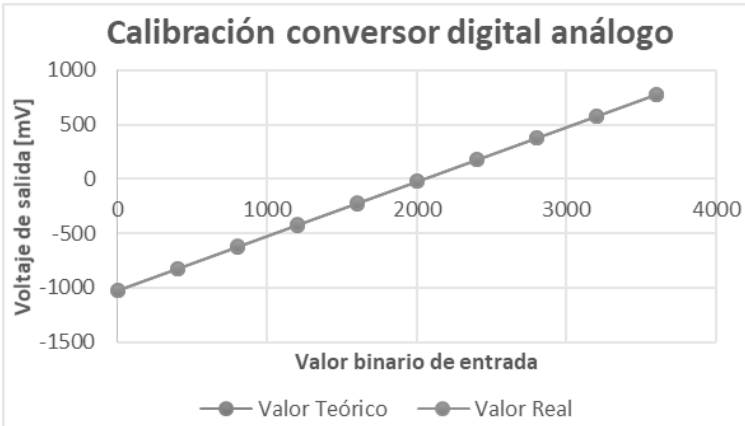

Fig. 13. Gráfica de comparación del valor teórico y el valor real de la salida del DAC.

Se observa una respuesta lineal del conversor digital análogo y un error menor a $5 \mathrm{mV}$. El error en la salida del conversor digital análogo, con respecto al valor teórico, se soluciona por software. Ya que, el DAC tiene una resolución de $0.5 \mathrm{mV}$. El factor de corrección es almacenado en la memoria EEPROM del microcontrolador y usado para corregir la salida del DAC.

La calibración del conversor análogo digital se realizó midiendo el voltaje de entrada en uno de los canales diferenciales del ADC. Se comparó la salida binaria teórica con la real. La tabla 5 y la figura 14 muestran los resultados obtenidos.
Tabla 5. Calibración conversor análogo digital.

\begin{tabular}{|lclll|}
\hline $\begin{array}{c}\text { Voltaje } \\
\text { de } \\
\text { entrada } \\
{[\mathrm{mV} \text { ] }}\end{array}$ & $\begin{array}{c}\text { Salida } \\
\text { ADC } \\
\text { teórica }\end{array}$ & $\begin{array}{c}\text { Salida } \\
\text { ADC } \\
\text { real }\end{array}$ & $\begin{array}{l}\text { Error } \\
-574,7\end{array} 16672$ & $\begin{array}{c}\text { Error } \\
{[\mathrm{mV}]}\end{array}$ \\
$-462,4$ & 19678 & 6 & 0,197 \\
$-349,6$ & 22977 & 22974 & -3 & $-0,1$ \\
$-236,5$ & 26144 & 26143 & -1 & $-0,049$ \\
-124 & 29295 & 29298 & 3 & 0,102 \\
$-11,5$ & 32446 & 32444 & -2 & $-0,069$ \\
100,7 & 35588 & 35592 & 4 & 0,133 \\
212,8 & 38728 & 38732 & 4 & 0,148 \\
325,2 & 41876 & 41879 & 3 & 0,113 \\
437,26 & 45014 & 45008 & -6 & $-0,224$ \\
\hline
\end{tabular}

\section{Calibración medidor de corriente}

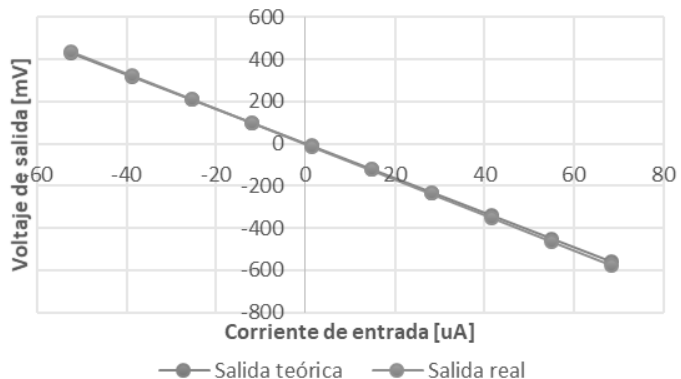

Fig. 14. Gráfica de comparación del valor teórico y el valor real de la salida del conversor análogo digital.

las pruebas anteriores evidencian un correcto funcionamiento del transductor amperométrico. Los errores obtenidos son bajos y corregibles por software. La linealidad, precisión y bajo ruido del circuito, hacen de este una excelente herramienta para la medición de las corrientes bajas, generadas en el proceso de amperometría.

Se realizó la prueba de amperometría, usando AChE (acetilcolinesterasa), un biomediador enzimático que reacciona a la presencia del Clorpirifos (figura 15). 


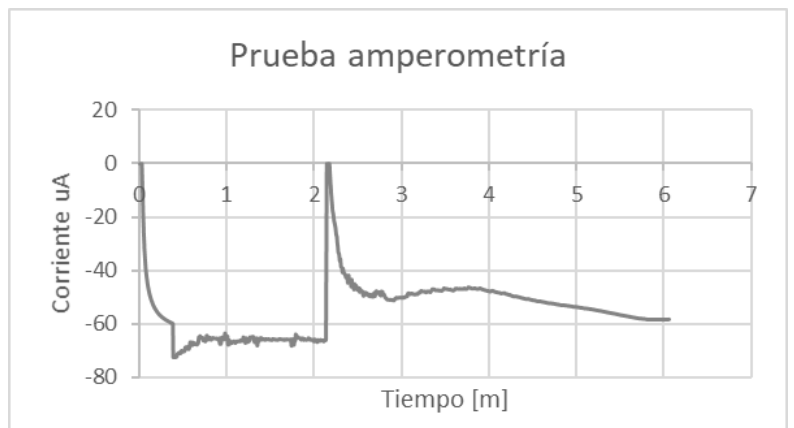

Fig. 15. Prueba amperometría biosensor enzimático

La prueba se realizó añadiendo a la muestra de agua $0.5 \mathrm{ug} / \mathrm{L}$ de clorpirifos. Al minuto $2: 20$ se puso en contacto la muestra de agua con el biosensor, generando un porcentaje de inhibición del 12\%, dando positivo para la detección de contaminante.

Además, se realizaron pruebas utilizando diferentes concentraciones del contaminante, en electrodos de diferentes materiales: electrodos de carbono, electrodos de carbono-ferrocianuro y electrodos de carbono-prussian blue. El ferrocianuro y el prussian blue, son aditivos que se añaden al carbono para intensificar la reacción de oxido/reducción, obteniendo una mayor intensidad de corriente en las pruebas de amperometría. Los resultados de estas pruebas se observan en la figura 16.

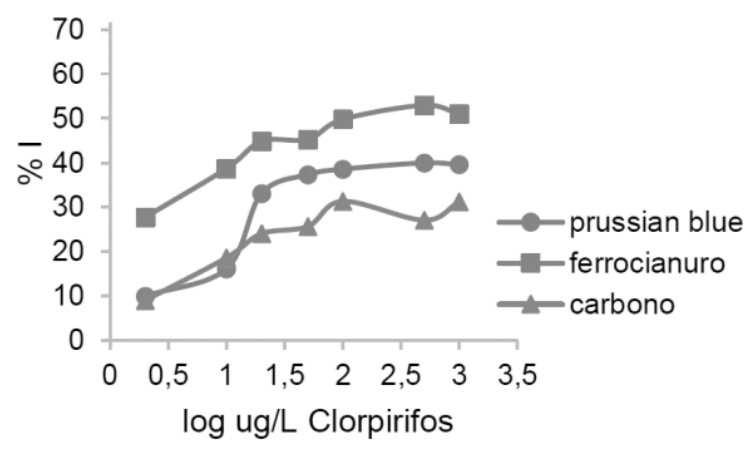

Figura 16. Ensayos de inhibición del clorpirifos solución acuosa.

En esta prueba se evidencia el correcto funcionamiento del biosensor enzimático utilizado. Además, se puede concluir que los biosensores no tienen una respuesta lineal a la concentración del analito, por lo que su uso es cualitativo y no cuantitativo, permitiendo detectar la presencia del analito, pero no su concentración exacta.

\section{Unidad central de procesamiento}

Se realizaron pruebas del consumo de energía de las dos versiones de Raspberry $\mathrm{Pi}$, corriendo los dos sistemas operativos oficiales (figuras 17 y 18). Se observó que para las mismas condiciones de procesamiento la Raspberry Zero $\mathrm{W}$ tuvo un consumo de aproximadamente la mitad del consumo de la Raspberry Pi 3B. Además, de los dos sistemas operativos el Raspbian Lite fue el que menor consumo energético requirió.

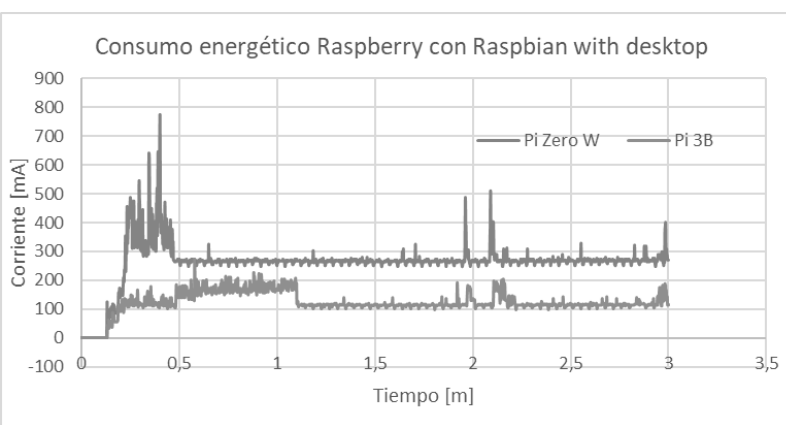

Figura 17. Consumo de energía Raspberry Pi con Rasbian with desktop

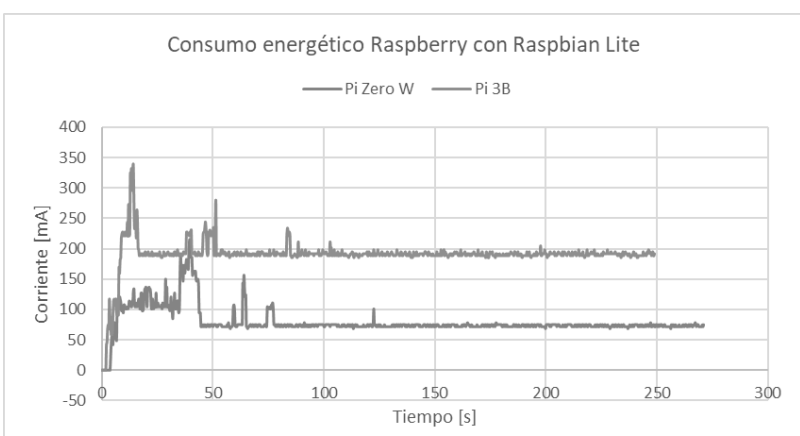

Figura 18. Consumo de energía Raspberry Pi con Rasbian Lite.

También se pudo observar que el tiempo de arranque de la Raspberry $\mathrm{Pi}$ Zero $\mathrm{W}$, es aproximadamente el doble que el de la Raspberry Pi 3B. Sin embargo, este tiempo de arranque es relativamente bajo, teniendo en cuenta que el periodo de muestreo de cada nodo de la red es de una hora. Por lo tanto, para la unidad de control de cada nodo de la red, se usa una Raspberry Pi Zero W.

Se ensamblaron tres nodos de medida para realizar la prueba de funcionamiento de la red inalámbrica. Cada nodo de medida consiste en dos 
transductores y una unidad de procesamiento central (figura 19 y 20).

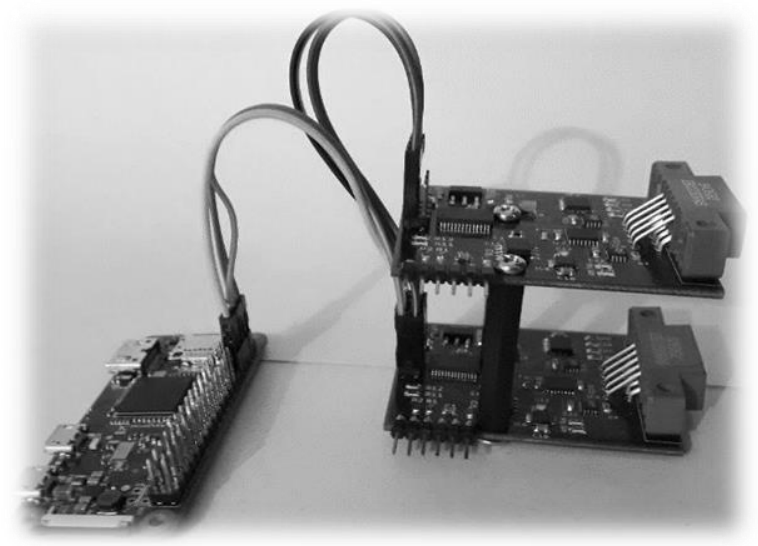

Fig. 19. Nodo de medida de la red inalámbrica

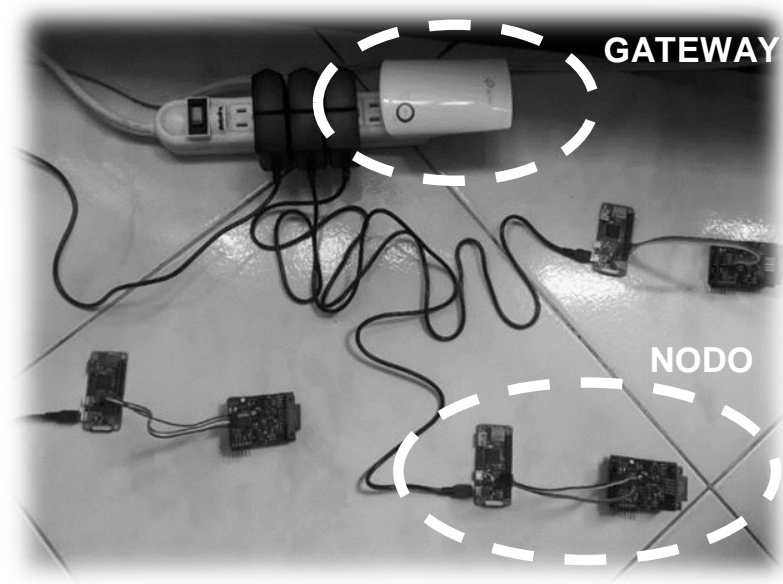

Fig. 20. Red inalámbrica de tres nodos de medida

\section{CONCLUSIÓN}

Los resultados obtenidos tanto a nivel de Hardware, como de software y su validación, han permitido que el sistema implementado pueda ser aplicado en con diferentes biosensores y permite ampliar su aplicación a otro tipo productos, ampliando a su vez, la capacidad de detección de contaminantes, simplemente agregando a la red más nodos y sensores.

La estructura de la red garantiza independencia entre los nodos, es decir, en caso de falla de alguno de los nodos, los restantes pueden seguir comunicándose con el Gateway. Evitando así la perdida masiva de información.
Como trabajos futuros se pueden desarrollar transductores para biosensores fuorescentes, este tipo de biosensores también es utilizado en la detección de contaminantes y pueden ser añadidos a los nodos de medida para tener diferentes tipos de biosensores y ampliar la capacidad de detección de la red. Además, se puede desarrollar un sistema de alimentación basado en energías renovables. Al desarrollar el sistema basado en energía solar y/o eólica, es posible instalar la red de biosensores en lugares remotos, sin tener que preocuparse por la carga de las baterías que alimentan a los nodos de medida.

\section{AGRADECIMIENTOS}

Los autores agradecen a Colciencias por la financiación del proyecto con código 111571451059 (contrato 147-2016) de la convocatoria 714-2015 Inv. Desarrollo Tecn. e Innovación en Ambiente, Océanos y Biodiversidad.

\section{REFERENCIAS BIBLIOGRÁFICAS}

[1] G.Pezzotti, A.Tibuzzi, T.Lavecchia, G.Rea, A.Serafini, M.T.Giardi. Portable instruments based in multiband fluorescence transducers for biosensing applications. Revista Politecnica, Year 3, $n^{\circ}$ 5, pag 123- 128 Medellin, December 2007 ISSN 1900-2351.

[2] K. Buonasera, A. Gabrielli, G. Pezzotti Escobar, R. Briancesco, S. Paduano, M. Semproni, L. Bonadonna. Rapid detection of Escherichia coli in marine water samples by remote optical biosensor system. The 12th Workshop on Biosensors \& BioAnalytical Microtechniques in Environmental, Food \& Clinical Analysis, ISBN 9788880802457, September 2017, Rome, Italy.

[3] G.Pezzotti, K . Buonasera, I. F. Pezzotti, J.B. Cano. Device and method for detecting biological contaminants, in particular coliform bacteria in water samples. PCT.IB2016.055263 WIPO, September 2016.

[4] K. Buonasera, G. Pezzotti Escobar, A. Gabrielli, A. Nicolosi, R. Briancesco, S. Paduano, M. Semproni, L. Bonadonna. A Completely Automatic 
Optical Biosensor System for Stressed Bacteria In Marine Water. Book XV IHSS Pharmacological Research And Analytical Approaches, ISBN 9788867416592, Pisa University Press, October, 2016, Italy.

[5] K. Buonasera, J. Griffiths, D. Hernández, T. Noguer, T. Ziemann, G. Pezzotti. A new biosensor for the screening of toxic compounds in water samples. 2nd International Conference on Biosensing Technology, October 10-12, 2011, Amsterdam, The Netherlands

[6] M. Turemis, S. Silletti, G. Pezzotti, J. Sanchís, M. Farré, M T. Giardi, L. Moro. Optical biosensor based on the microalga-paramecium symbiosis for improved marine monitoring. Sensors and Actuators B: Chemical (ISSN 0925-4005). Elsevier Science. April 2018. https://doi.org/10.1016/j.snb.2018.04.111.

[7] L. Moro, G. Pezzotti; M. Turemis; J. Sanchís; M. Farré; R. Denaro; M. G. Giacobbe; F. Crisafi; M T. Giardi. Fast Pesticide Pre-Screening in Marine Environment Using a Green Microalgae-based Optical Bioassay. Marine Pollution Bulletin 129 (2018) 212-221 Elsevier Science February 2018, (ISSN 0025-326X). DOI: 10.1016/j.marpolbul.2018.02.036.

[8] M.T.Giardi, M.Lambreva, G.Rea, A.Antonacci, A.Serafini, G.Pezzotti, T.Lavecchia. Computational and biotechnological approaches for the construction of enviromental biosensors for herbicide analyses. Environmental and Health Biosensor, Roma, 9-10 October 2007, ISPESL.

[9] J.Cano, K.Buonasera, G.Pezzotti, M.T.Giardi. New platform of biosensors based on fluorescence enviromental applications. Revista Politécnica, Politécnico Colombiano JIC. ISSN 1900-2351, P 124-132, year 3, №13 2011, Medellín.

[10] K.Buonasera, G.Pezzotti, I.Pezzotti, J.Cano, MTGiardi. Biosensors: new frontiers for the environmental analysis. Revista Politecnica, Politecnico Colombiano JIC. ISSN 1900-2351, P 93-100, year 3, №13 2011, Medellín.

[11] K.Buonasera, I.Pezzotti, G.Rea, G.Pezzotti, M.T.Giardi - "Design of a portable instrument for environmental applications by amperometric measurements on biological material" Revista Politecnica, Politecnico Colombiano JIC. Year 5, $n^{\circ}$
9, pag 107- 114 Medellín, December 2009 ISSN 1900-2351.

[12] M.T.Giardi, V.Scognamiglio, G.Rea, G. Rodio, A.Antonacci, M.Lambreva, A.Tibuzzi, G.Pezzotti, U.Johanningmeier. Optical biosensors for environmental monitoring based on computational and biotechnological tools for engineering the photosynthetic D1 protein of Chlamydomonas reinhardtii. Biosensor and Bioelectronics, Volume 25 issue 2, pages 294-30, Elsevier, 15 October 2009; (ISSN): 0956-5663, DOI: 10.1016/j.bios.2009.07.003.

[13] J. Cano, D. Giannini, G. Pezzotti, G. Rea, M.T. Giardi. Space Impact and Technological Transfer of a Biosensor Facility to Earth Application for Environmental Monitoring. Recent Patents on Space Technology, 2011, Volume 1, No. 1, March 15, 2011; ISSN 2210-6871; Bentham Science Publishers.

[14] G.Pezzotti, M.T.Giardi, M.Lambreva, G.Rea, I.Pezzotti. Development of new environmental biosensors instruments for herbicides analyses. Revista Politecnica, Year 4, $\mathrm{n}^{\circ}$ 6, pp. 116- 123 Medellin, January 2008 ISSN 1900-2351.

[15] J.B. Cano Quintero, K. Buonasera, G. Pezzotti, M.T. Giardi. Multichannel fluorimeter for biosensor applications on environmental field. pag 79-83, Lecture Notes in Electrical Engineering,Sensors , Springer New York 2013, ISSN 1876-1100,18761119 doi: 10.1007/978-1-4614-3860-1_12.

[16] M.T.Giardi, M.Lambreva, G.Rea, A.Antonacci, A.Serafini, G.Pezzotti, T.Lavecchia. Computational and biotechnological approaches for the construction of enviromental biosensors for herbicide analyses. Enviromental and Health Biosensor, 10th October 2007, ISPESL, Rome, Italy.

[17] V.Scognamiglio, G.Pezzotti, I.Pezzotti, J.Cano, K.Buonasera, D.Giannini, M.T.Giardi. Biosensors for Effective Environmental and Agrofood Protection and Commercialization: from research to market. Microchimica Acta, SpringerLink ISSN 0026-3672, Jan 2010., DOI: 10.1007/s00604-010-0313-5.

[18] R. Penu, S. C. Litescu, S. A.V. Eremia, G.L. Radu, M.T. Giardi, G. Pezzotti, G. Rea. Application of an optimized electrochemical sensor in astaxanthin antioxidant properties monitoring 
against lipoperoxidation, during algae accumulation. New Journal of Chemistry - The Royal Society of Chemistry 2013, (ISSN): 1144-0546, DOI: $10.1039 / \mathrm{C} 5 \mathrm{NJ} 00457 \mathrm{H}$.

[19] A.Tibuzzi, G.Pezzotti, G.Rea, D.Bianconi, M.T.Giardi. A RC-based biosystem for measuring anti-oxidizing power. Technical Proceedings of the 2007 NSTI Nanotechnology Conference and Trade Show, Volume 2, Chapter 6: Biosensors, pp. $519-$ 522, ISBN:1-4200-6183-6.

[20] V.Scognamiglio, G.Pezzotti, I.Pezzotti, J.Cano, I.Manfredonia, K.Buonasera, F. Arduini, D. Moscone, G. Palleschi, M.T.Giardi. Towars an integrated Biosensors array for simultaneous and rapid multi-analisys of endocrine disrupting chemicals. Analytica Chimica Acta, Elsevier Science, 2012, (ISSN): 0003-2670, DOI: 10.1016/j.aca.2012.09.010.

[21] G.Pezzotti, J.Cano, K.Buonasera. Biosensing technologies for space applications. Revista Politecnica, Politecnico Colombian JIC. ISSN 19002351, P 133-143, year 3, №13 2011, Medellin, Colombia.

[22] M.Turemis, M.T.Giardi, G.Pezzotti. Space impact of a biosensor facility for water control in ISS and technological transfer to Earth applications. workshops "Bioregenerative Life Support" of International Space Life Sciences Working GroupISLSWG, Turin, 18-19 May 2015.

[23] V.Scognamiglio, D.Raffi, M.Lambreva, G.Rea, A.Tibuzzi, G.Pezzotti, U. Johanningmeier, M.T.Giardi. Chlamydomonas reinhardtii genetic variants as probes for fluorescence sensing system in detection of pollutants. Analytical and Bioanalytical Chemistry, Springer Berlin / Heidelberg ISSN1618-2642 394 (4), p.1081-1087, Jun 2009.

[24] I.Manfredonia, M.Barbieri, R.Verzicco, C.Stallo, M.Ruggieri, T.Lavecchia, M.Turemis, G.Pezzotti "Performance Analysis for Steady Flow Generation and Improved Readout Signal in Amperometric Biosensors", IEEE Sensors Journal Sensors 11214-2014, 2015 (ISSN 1530-437X)

[25] A.Tibuzzi, G.Pezzotti, T.Lavecchia, G.Rea, M.T.Giardi. A Portable Light-Excitation Equipped Bio-Amperometer for Electrogenic Biomaterials to Support the Technical Development of Most
Biosensors. Sensors \& Transducers Journal, Volume 88, Issue 2, ISSN 1726-5479 February 2008.

[26] A.Tibuzzi, G.Rea, G.Pezzotti, D.Esposito, U.Johanningmeier, M.T.Giardi. A new miniaturized multiarray biosensor system for fluorescence detection. Journal of Physics: Condensed Matter, vol. 19 Issue 39, 395006, ISSN 0953-8984, 2007.

[27] M.Lambreva, G.Pappalettera, A.Palasciano, P.Boffi, V.Scognamiglio, G.Pezzotti, M.T.Giardi. Experimental Laser-induced fluorescence sensor for remote detection of herbicide action on plants. XIII International Symposium on Luminescence Spectrometry, Analytical luminescence (ISLS 2008), John Wiley \& Sons, Vol. 23, Issue 4, pp.191-280, July 2008, Bologna, Italy.

[28] G.Pezzotti, M.T.Giardi, G.Rea, A.Tibuzzi, M.Lambreva. Portable instrument based in biosensoristic systems for applications with biological material. 1st International symposium on instrumentation and industrial control, Memoria, Numero 38, ISBN 16920368, ITM, April 2008, Medellin, Colombia.

[29] G.Pezzotti, A.Tibuzzi, T.Lavecchia, G.Rea, M.T.Giardi. Portable bio-amperometer for photoactive biomaterial monitoring. 13th Italian Conference on Sensors and Microsystems AISEM 2008 - XIII Conferenza Annuale, 19 - 21, February 2008, Rome, Italy ISBN 978-981-283-597-0.

[30] J.B. Cano, K. Buonasera, G. Pezzotti "Transduction methods used on biosensors: amperometry and fluorescence" Rev. Fac. Ing. Univ. Antioquia N. 72, pp. 104-115, 2014 (ISSN 0120-6230).

[31] K.Buonasera, G.Pezzotti, V.Scognamiglio, A.Tibuzzi, M.T.Giardi. New Platform of Biosensors for Prescreening of Pesticide Residues To Support Laboratory Analyses. Journal Agricultural and Food Chemistry. 58, 5982-5990; of American chemical society 2010, (ISSN): 0021-8561.

[32] I. Manfredonia, C. Stallo, M. Ruggieri, K. Buonasera, G. Pezzotti.. Architecture design of an early warning monitoring system for chemical water bodies contamination. Conference: 4th International Conference on Wireless Communications, Vehicular Technology, Information Theory and Aerospace \& Electronic Systems (VITAE) Location: 
Aalborg, Denmark May 2014, ISBN: 978-1-47994626-6.

[33] B. Bucur, F.D. Munteanu, J. L. Marty, A. Vasilescu. Advances in enzyme-based biosensors for pesticide detection. Biosensor, 8(2). 2018.

[34] M. Zennaro, I. C. T. P. Trieste-Italy. Introduction to Wireless Sensor Networks. 2012.

[35] R. Hidalgo, J. I. Moreno Routing Design in Wireless Sensor Networts and Solution for Healthcare Environment. IEEE Latin Am. Trans, 9. 2011.

[36] Ali, H., Soe, J. K., \& Weller, S. R. A real-time ambient air quality monitoring wireless sensor network for schools in smart cities. In Smart Cities Conference (ISC2), IEEE First International (pp. 16). 2015.

[37] Lara, R., Benítez, D., Caamaño, A., Zennaro, M., \& Rojo-Álvarez, J. L. On real-time performance evaluation of volcano-monitoring systems with wireless sensor networks. IEEE Sensors Journal, 15(6), 3514-3523. 2015.

[38] Polo, J., Hornero, G., Duijneveld, C., García, A., \& Casas, O. Design of a low-cost Wireless Sensor Network with UAV mobile node for agricultural applications. Computers and electronics in agriculture, 119, 19-32. 2015.

[39] Vikram, N., Harish, K. S., Nihaal, M. S., Umesh, R., Shetty, A., \& Kumar, A. A low-cost home automation system using wi-fi based wireless sensor network incorporating Internet of Things (IOT). In Advance Computing Conference (IACC), IEEE 7th International (pp. 174-178). 2017.

[40] Raspberry Pi Foundation. Disponible en: https://www.raspberrypi.org/. [consultado el 15 de noviembre de 2018].

[41] Electrodos serigrafiados Metrohm DropSens, Disponible en: http://www.dropsens.com/screen printed electrode s_pag.html. [consultado el 24 de noviembre de 2018]. 
